\title{
Noninvasive Cardiac Assessment in Children of Women with Systemic Lupus Erythematosus
}

\author{
Maria de Fátima Monteitro Pereira Leite, Roger Aaron Levy, Mônica Scott Borges, \\ Eduardo Correa Barbosa, Paulo Ginefra, Paulo Roberto Barbosa Benchimol, \\ Nadia Barreto Tenório Aoun, Luiz Alberto Christiani, Francisco Manes Albanesi Filho
}

Rio de Janeiro, RJ - Brazil

\begin{abstract}
Objective - Noninvasive cardiac assessment of newborns and infants of women with systemic lupus erythematosus. The children had no congenital total atrioventricular block and were compared with the children of healthy women.
\end{abstract}

Methods - We prospectively assessed 13 newborns and infants aged 1 to 60 days, children of women with systemic lupus erythematosus and without congenital total atrioventricular block. These children were compared with 30 children of women who had no lupus or anti-Ro/ SSA antibodies, and no risk factors for congenital heart disease either. Their age groups matched. The following examinations were performed: cardiological physical examination, electrocardiography, echocardiography, and signal-averaged electrocardiography.

Results - The statistical analysis showed no significant difference in ventricular function or in the cardiac conduction system between the groups.

Conclusion - In regard to the conduction system and ventricular function in the absence of total atrioventricular block, no statistically significant difference was observed between the children of women with systemic lupus erythematosus and children of healthy women.

Key words: total atrioventricular block, neonatal lupus, congenital arrhythmia

Hospital Universitário Pedro Ernesto - UERJ

Mailing address: Maria de Fátima M. P. Leite - Rua Magalhães Couto, 784/34

Cep 20735-180 - Rio de Janeiro, RJ, Brazil - E-mail: albanesi@ cardiol.br

Received 10/27/02

Accepted 1/14/03

Englis version by Stela Maris C. e Gandour
Neonatal lupus is an autoimmune disease due to passive acquisition of maternal antibodies during pregnancy ${ }^{1,2}$. These antibodies remain in the fetal and newborn circulation causing skin, hematological, and cardiologic abnormalities, and other conditions that imitate adult systemic lupus erythematosus ${ }^{1,3,4}$. These abnormalities are transient if in tissues capable of continuous regeneration, such as the skin and the hematopoietic system, and irreversible in the cases of impairment of the cardiac conduction system ${ }^{1}$.

In healthy pregnant women, the frequency of anti-Ro/ SSA antibodies through immunodiffusion or contraimmunoelectrophoresis varies around 0.4 and $1 \%$, respectively, and of anti-La/SSB antibodies around $0.7 \%{ }^{5}$. In patients with systemic lupus erythematosus, the frequency of antiRo/SSA antibodies is approximately $40 \%$ and that of anti$\mathrm{La} / \mathrm{SSB}$ antibodies is 15 to $20 \%{ }^{5}$. In patients with Sjögren's syndrome, the frequency of anti-Ro/SSA antibodies is 40 to $45 \%$ and that of anti-La/SSB antibodies is 15 to $20 \%{ }^{5}$.

The general prevalence of neonatal lupus in newborns of mothers with systemic lupus erythematosus ranges from 1 to $3 \%{ }^{5}$, and the general estimate of a child with congenital total atrioventricular block is $1: 60$, which increases to $1: 20^{9}$ if the mother has circulating anti-Ro/SSA antibodies ${ }^{5-8}$.

In 1993, Goble et al $^{8}$ reported that newborns from mothers with systemic lupus erythematosus who developed the disease before or during pregnancy had an increase in heart rate (corrected for age) and in the PR interval in the electrocardiogram corrected for heart rate. Other disorders of cardiac electrical conduction in children of women with anti-Ro/SSA antibodies have been reported, and the classification of these disorders as manifestations of neonatal lupus is still controversial ${ }^{9,10}$.

This study aimed at comparing, from the cardiovascular point of view, children of women with systemic lupus erythematosus and children of healthy women through physical examination, electrocardiography, 2-dimensional Doppler echocardiography, color flow Doppler, and signalaveraged electrocardiography. 


\section{Methods}

From March 1995 to July 1997, 13 newborns and infants from women with systemic lupus erythematosus (group I) were prospectively studied. Their ages ranged from 1 to 60 days, and 8 children were males. All mothers diagnosed with the rheumatologic disease had it confirmed through clinical and laboratory means, and 9 were positive for the anti-Ro/SSA antibodies according to the guidelines of the American Society of Rheumatology ${ }^{11}$. The control group (group II) was composed of healthy children in the same age bracket whose mothers neither had nor were suspected of having systemic lupus erythematosus or Sjögren's syndrome, with confirmation of this through clinical and laboratory means. The mothers of group II children were negative for anti-Ro/SSA and anti-La/SSB antibodies in a blood sample collected when their children were assessed.

The procedures involved in the study were explained to all mothers, who agreed with them through written informed consent.

The children studied underwent a physical examination especially directed at the cardiovascular system and the following complementary tests: conventional 12-lead electrocardiography, 2-dimensional Doppler echocardiography, color flow Doppler, and signal-averaged electrocardiography. The Capurro score was not considered a limitation for inclusion in the control group, in which premature babies as young as 35 gestational weeks were accepted as long as neonatal complications were not present, for matching the gestational age of group I. A Capurro score lower than 38 weeks was considered a prematurity criterion. The criterion for asphyxia was an Apgar score below 7 in the fifth minute of life. During the study, 3 cases of congenital total atrioventricular block were observed and excluded from the statistical analysis.

The electrocardiogram was obtained at rest and without sedatives. The following parameters were analyzed: rhythm, heart rate, electric axis ( $\hat{A} Q R S$ ), amplitude of the $\mathrm{R}$ wave in $\mathrm{V}_{1}$ $\left(\mathrm{RV}_{1}\right)$, and the QT interval corrected for heart rate (QTc).

In the echocardiogram, the following parameters were measured in millimeters $(\mathrm{mm})$ : the diameters of the aorta (AO), of the left atrium (LA), of the left ventricle at the end of diastole (EDLVD) and systole (SLVD), of the right ventricle at the end of diastole (RV), diastolic thickness of the interventricular septum (IVS) and of the left ventricular posterior wall (LVPW). Left ventricular ejection fraction (EF) and systolic shortening fraction (SF), used to assess ventricular function, were analyzed. All measures were taken according to the recommendations of the American Society of Echocardiography ${ }^{12}$. Based on the values obtained on echocardiography, left ventricular mass (LVM) was determined in grams $(\mathrm{g})$ with the formula $\mathrm{LVM}=0.832[(\mathrm{IVS}+\mathrm{LVPW}+$ $\left.\mathrm{EDLVD}^{3}-(\mathrm{EDLVD})^{3}\right]+0.6^{13}$.

The signal-averaged electrocardiogram was obtained using the Predictor IIc system (ART-Austin, Texas, USA). Medi-Trace silver electrodes of the pediatric size or modeled to adapt to the child's thoracic surface were used and placed in the modified Frank XYZ leads, according to the follo- wing criteria: X lead - 4th intercostal space, right and left midaxillary lines, positive electrode to the left; Y lead-left midclavicular line, infraclavicular, and left costal margin, the positive electrode being that in the costal margin; $\mathrm{Z}$ lead at the level of the $\mathrm{V}_{2}$ lead of the conventional electrocardiogram, with its representation in the left paravertebral line, the anterior electrode being the positive.

The signal was considered adequate when the noise at the beginning of the capturing process was below $5 \mathrm{mV}$ in all leads. In children with an initial noise greater than $5 \mathrm{mV}$, the skin was prepared again at the site of the impaired lead.

Considering the arithmetic mean of the 3 leads, the noise was reduced to a level $\leq 0.5 \mathrm{mV}$, which was automatically obtained by the capturing system. The signals were captured in the sampling frequency of $2000 \mathrm{~Hz}$, with the fiducial point shifted to the 400 position. The QRS complex was maintained as the region for comparison with the default parameters of the equipment.

The noise window was positioned in a region of the signal away from the QRS and P-wave deflections, usually in the region of the ST segment.

The analysis of the signals was divided into the following 2 steps: time domain and frequency domain.

Time domain - To assess ventricular activation, each lead underwent a filtering process with the Butterworth bidirectional 4-pole filter with a high-pass cut-off frequency of $40 \mathrm{~Hz}$ and a low-pass cut-off frequency of $250 \mathrm{~Hz}$. The 3 leads filtered were combined in a vectorial resultant, the magnitude vector (MV), using the formula: $\mathrm{VM}=\sqrt{ } \mathrm{X}^{2}+\mathrm{Y}^{2}+$ $\mathrm{Z}^{2}$. The following variables were studied: total duration of the filtered QRS (DQRS); mean square root of tension of the final 40 ms (MSR40) and 20 ms (MSR20) of the filtered QRS; and total duration of the potentials with amplitude $\leq 40 \mathrm{mV}$ in the terminal region of the filtered QRS (LAS40), whose beginning and end were visually established after the automatic detection performed by the equipment.

The PR interval was measured in each nonfiltered X, Y, and $Z$ lead through visual identification of the beginning of the $\mathrm{P}$ and $\mathrm{Q}$ or R waves of the QRS complex. The point of the lesser visual curvature at the moment of junction of the baseline with the ascension of the $\mathrm{P}$-wave deflection, in which the tension of the base line did not vary in the preceding $2 \mathrm{~ms}$, was considered the beginning of the $\mathrm{P}$ wave. The beginning of the $\mathrm{Q}$ or $\mathrm{R}$ waves was identified as the point of lesser visual curvature at the moment of junction of the baseline with the first deflection of the QRS complex. Because of the rapid transition of the PR segment to ventricular activation (QRS complex), the point that was visually preceded by a region of little inclination and whose amplitude remained stable in the 2 preceding points was used. Of the 3 leads, the one whose PR interval was longer was used.

Frequency domain - This domain was used to analyze the QRS complex. The analysis was carried out with a timefrequency map, based on the Fourier transform applied to segments of short duration of the first derivative of the signal (SFFT). The time-frequency map was constructed as a module of the sum option of the acquisition system, each 
segment being multiplied by a compensation window of the Blackman Harris type to avoid discontinuity in the extremes of the interval. The point preceding the beginning of the filtered QRS (initial point of the map) in $25 \mathrm{~ms}$ was used. For calculating the spectral components, the window was shifted every $2 \mathrm{~ms}$ within a region of $200 \mathrm{~ms}$ from the initial point in the map. The 25-ms segment FFT was added to values ranging from zero (zero padding) to 64 points for the construction of the range of each window.

The variables used in the analysis of the time-frequency map were as follows: mean (Cem) and standard deviation (Cesd) of the intersegmentary spectral correlation of the electric signal throughout ventricular activation; mean $(\mathrm{Bdm})$ and standard deviation (Bdsd) of the frequency band delimitating the signal energy. The beginning and end of the QRS in the time-frequency map for analysis of the variables were visually detected. The other parameters of analysis were maintained in the default mode of the device.

Laboratory tests - Blood samples of all patients underwent the 2 following laboratory tests: FANA (fluorescent antinuclear antibody) (Biorad), and anti-Ro/SSA antibody by hemagglutination (Hemagen). Values greater than 1/50 were considered positive.

In regard to the statistical analysis, the nonparametric Mann-Whitney test (2-tailed) was used. The statistical calculation was performed with the aid of electronic computing with SPSS software for Windows. The significance level adopted was $\mathrm{P}<0.05$.

\section{Results}

The patients in both groups studied had a normal physical cardiac examination. Their ages and anthropometric measures showed no statistically significant differences.

The 12-lead surface electrocardiogram showed a significantly more elevated heart rate in group I as compared with that in group II. The other parameters studied were very similar (tab. I), heart rate being the only significantly different variable between the 2 groups, being greater in the children of women with systemic lupus erythematosus. The results showed no difference, even when considering the group of women with anti-Ro/SSA antibodies and the group of healthy women (tab. II).

No statistically significant difference was observed in regard to the dimensions of the cavities (tab. III). Analyzing left ventricular function, group I had significantly greater ejection fraction values $(76.77 \times 72.53 ; \mathrm{P}=0.049)$ than group II did (tab. III). No difference was found between the left ventricular masses in the 2 groups. When the subgroup comprised of women positive for anti-Ro/SSA antibodies was separated, the difference in the ejection fraction disappeared, and only the difference in the thickness of the left ventricular posterior wall was significant (tab. IV).

The values obtained with the signal-averaged electrocardiogram showed no significant difference in the QRS analysis and in the time and frequency domains (tab. V). The result did not change when only the subgroup of women with anti-Ro/SSA antibodies was assessed (tab. VI).

\begin{tabular}{|c|c|c|c|}
\hline & Group I ( $\mathrm{n}=13$ ) & Group II (n=30) & $P$ \\
\hline $\mathrm{HR}$ & 137.6 & 125.9 & 0.04 \\
\hline ÂQRS & 120.4 & 130.7 & 0.20 \\
\hline $\mathrm{RV}_{1}$ & 20 & 21.7 & 0.75 \\
\hline QTc (ms) & 40 & 39 & 0.20 \\
\hline
\end{tabular}

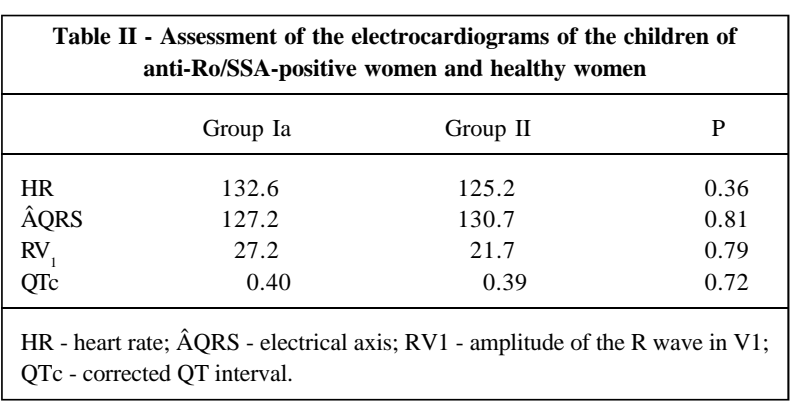

\begin{tabular}{|c|c|c|c|}
\hline \multicolumn{4}{|c|}{$\begin{array}{l}\text { Table III - Values of the measures obtained on the echocardiogram } \\
\text { (means of the diameters and mass, and assessment of ventricular } \\
\text { function) in the groups studied }\end{array}$} \\
\hline & Group I ( $n=13)$ & Group II $(\mathrm{n}=30)$ & $P$ \\
\hline $\mathrm{AO}(\mathrm{mm})$ & 8.7 & 9 & 0.30 \\
\hline $\mathrm{LA}(\mathrm{mm})$ & 12.3 & 11.7 & 0.49 \\
\hline $\operatorname{SLVD}(\mathrm{mm})$ & 10.8 & 11.5 & 0.30 \\
\hline $\operatorname{EDLVD}(\mathrm{mm})$ & 17.5 & 17.9 & 0.30 \\
\hline $\mathrm{RV}(\mathrm{mm})$ & 6.7 & 6.6 & 0.84 \\
\hline IVS (mm) & 3.5 & 3.4 & 0.43 \\
\hline LVPW (mm) & 3.1 & 3.4 & 0.14 \\
\hline $\mathrm{EF}(\%)$ & 76.8 & 72.5 & 0.049 \\
\hline $\mathrm{SF}(\%)$ & 38.6 & 35.7 & 0.07 \\
\hline $\operatorname{LVM}(\mathrm{g})$ & 8.2 & 8.7 & 0.44 \\
\hline \multicolumn{4}{|c|}{$\begin{array}{l}\text { Ao- diameter of the aorta; LA- diameter of the left atrium; EDLVD- end-diastolic } \\
\text { left ventricular diameter; SLVD- systolic left ventricular diameter; RV- end- } \\
\text { diastolic right ventricular diameter; IVS- diastolic thickness of the interven- } \\
\text { tricular septum; LVPW- thickness of the left ventricular posterior wall during } \\
\text { diastole; EF- left ventricular ejection fraction; SF- systolic shortening fraction; } \\
\text { LVM- left ventricular mass. }\end{array}$} \\
\hline
\end{tabular}

The PR interval showed no significant difference between the 2 groups, even when considering the subgroup of women positive for anti-Ro/SSA antibodies alone.

\section{Discussion}

One of the most severe forms of presentation of neonatal lupus is the one that includes congenital total atrioventricular block ${ }^{14}$. Several attempts have been made to demonstrate that, even in the absence of that block, cardiological manifestations of lesser clinical significance exist, indicating some degree of myocardial impairment in these patients ${ }^{8,10}$. Our study showed some differences between groups I and II; however, those with statistical significance revealed only small differences between the groups and did 


\begin{tabular}{|c|c|c|c|}
\hline \multicolumn{4}{|c|}{$\begin{array}{l}\text { Table IV - Measures obtained on the echocardiogram (means of the } \\
\text { diameters and mass, and assessment of ventricular function) in the } \\
\text { children of anti-Ro/SSA-positive women without congenital total } \\
\text { atrioventricular block compared with those in group II }\end{array}$} \\
\hline & Group Ia & Group II & $\mathrm{P}$ \\
\hline $\mathrm{AO}(\mathrm{mm})$ & 8.9 & 9 & 0.43 \\
\hline $\mathrm{LA}(\mathrm{mm})$ & 12.1 & 11.7 & 0.63 \\
\hline $\operatorname{SLVD}(\mathrm{mm})$ & 10.4 & 11.5 & 0.20 \\
\hline $\operatorname{EDLVD}(\mathrm{mm})$ & 16.9 & 17.9 & 0.36 \\
\hline $\mathrm{RV}(\mathrm{mm})$ & 6.9 & 6.6 & 0.66 \\
\hline IVS(mm) & 3.4 & 3.4 & 0.86 \\
\hline LVPW (mm) & 2.9 & 3.4 & 0.04 \\
\hline $\mathrm{EF}(\%)$ & 76.7 & 72.5 & 0.06 \\
\hline $\mathrm{SF}(\%)$ & 38.6 & 35.7 & 0.10 \\
\hline $\operatorname{LVM}(\mathrm{g})$ & 7.4 & 8.7 & 0.09 \\
\hline \multicolumn{4}{|c|}{$\begin{array}{l}\text { Ao- diameter of the aorta; LA- diameter of the left atrium; EDLVD- end-diastolic } \\
\text { left ventricular diameter; SLVD- systolic left ventricular diameter; RV- end- } \\
\text { diastolic right ventricular diameter; IVS- diastolic thickness of the interven- } \\
\text { tricular septum; LVPW- thickness of the left ventricular posterior wall during } \\
\text { diastole; EF- left ventricular ejection fraction; SF- systolic shortening fraction; } \\
\text { LVM- left ventricular mass. }\end{array}$} \\
\hline
\end{tabular}

Table V - Comparison of the means of QRS in the time and frequency

\begin{tabular}{|lccc|}
\multicolumn{4}{|c|}{ domains } \\
\hline & Group I (n=13) & Group II (n=30) & P \\
\hline DQRS (ms) & 55 & 56 & 0.57 \\
MSR20 (mv) & 177.8 & 139.5 & 0.41 \\
MSR40(mV) & 550 & 464.4 & 0.11 \\
LAS40 (ms) & 9.7 & 10.2 & 0.39 \\
Cem & 0.96 & 0.96 & 0.17 \\
Cesd & 0.06 & 0.05 & 0.31 \\
Bdm & 60.7 & 62.3 & 0.19 \\
Bdsd & 26.8 & 27.9 & 0.50 \\
PR & 187.5 & 120.7 & 0.84 \\
\hline
\end{tabular}

DQRS - total duration of the filtered QRS; MSR20 - mean square root of the tension of the final $20 \mathrm{~ms}$ of the filtered QRS; MSR 40 - mean square root of the tension of the final $40 \mathrm{~ms}$ of the filtered QRS; LAS40 - total duration of the potentials with amplitude $\leq 40 \mathrm{mV}$ in the terminal region of the filtered QRS; Cem - mean of the intersegmentary spectral correlation of the electric signal throughout ventricular activation; Cesd - standard deviation of the intersegmentary spectral correlation of the electric signal throughout ventricular activation; Bdm - mean of the frequency band delimitating the signal energy; Bdsd - standard deviation of the frequency band delimitating the signal energy; PR - PR interval measured on signal-averaged electrocardiogram.

not suggest cardiological impairment of the children due to maternal systemic lupus erythematosus. No impairment in ventricular function or electrocardiographic change that could confirm cardiac damage was observed in the neonatal period until the 60th day of life.

The 12-lead conventional electrocardiogram showed differences between the groups in regard to heart rate values, which were greater in group I (tab. I). Goble et al ${ }^{8} \mathrm{re-}$ ported similar results, which were also related to fetal distress. However, our case series shows that a greater heart rate in these patients is also accompanied by normal systolic and diastolic left ventricular diameters, normal ejection and shortening fractions, ruling out ventricular dysfunction as a cause of tachycardia. When the subgroup of the children of women with anti-Ro/SSA antibodies was separated, this

\begin{tabular}{|lccc|}
\hline \multicolumn{4}{|c|}{ Table VI - Assessment of signal-averaged electrocardiogram in the Ia } \\
(Anti-Ro/SSA +) and II groups
\end{tabular}

DQRS - total duration of the filtered QRS; MSR20 - mean square root of the tension of the final $20 \mathrm{~ms}$ of the filtered QRS; MSR 40 - mean square root of the tension of the final $40 \mathrm{~ms}$ of the filtered QRS; LAS40 - total duration of the potentials with amplitude $\leq 40 \mathrm{mV}$ in the terminal region of the filtered QRS; $\mathrm{Cem}$ - mean of the intersegmentary spectral correlation of the electric signal throughout ventricular activation; Cesd - standard deviation of the intersegmentary spectral correlation of the electric signal throughout ventricular activation; Bdm - mean of the frequency band delimitating the signal energy; Bdsd - standard deviation of the frequency band delimitating the signal energy; PR - PR interval measured on signal-averaged electrocardiogram.

difference disappeared, showing once again that the presence of anti-Ro/SSA antibodies was not the factor determining the greater heart rate found in the subgroup of women with those antibodies.

The echocardiographic measures, as expected, were similar, because the patients had neither signals nor symptoms of fetal or neonatal heart failure. However, a significantly greater ejection fraction was found in group I. The statistically significant differences related to ejection fraction may be justified by the fact that that parameter is a mathematical formula in which the systolic and diastolic left ventricular diameters are cubed. Thus, when cubed, small nonsignificant differences in ventricular diameters become statistically significant without denoting change in the real function. In addition, the ejection fraction was greater in the group studied, and no scientific explanation exists for the better ventricular function in the children of patients with systemic lupus erythematosus. Moreover, the shortening fraction, another parameter used to assess ventricular function, was similar between the groups, emphasizing once again the possibility that a greater ejection fraction is a bias due to the use of a mathematical resource that increases the difference between the values of left ventricular cavity diameters. Still considering the echocardiographic values, the differences disappeared when only the children of antiRo/SSA-positive women were assessed.

Although children of women with systemic lupus erythematosus have not been reported as candidates for ventricular arrhythmia, the use of signal-averaged electrocardiography is justified when evidence of myocardial inflammatory disease is searched for in these patients. Therefore, we could find changes in the spectral turbulence similar to those found in patients in the acute and subacute phases of myocarditis with other causes, in which signal-averaged electrocardiography was more effective than other conventional diagnostic methods to detect evidence for a myocardial inflammatory process ${ }^{16}$. Reports on signal-averaged 
electrocardiography in newborns and infants are scarce, ours being one of the first studies on this subject in our country ${ }^{16}$. The normal values of the signal-averaged electrocardiogram in the age bracket of the patients studied have not yet been standardized. That was the reason why the control group was carefully selected to include healthy and nonasphyxial newborns and infants, and mothers with normal prenatal follow-up and no risk factors for myocardial damage. The QRS assessment revealed very similar values in the 2 groups, and no pattern suggestive of spectral turbulence was found in the time and frequency domains.

The results obtained in the signal-averaged electrocardiogram suggest that the children of women with systemic lupus erythematosus have no changes in intramyocardial ventricular conduction. Although this does not definitively rule out the presence of myocarditis, it suggests at least that no detectable damage to ventricular intramyocardial conduction occurred during intrauterine life.

The PR interval was assessed on the signal-averaged electrocardiogram, and, although it was greater in absolute numbers in the children of women with systemic lupus erythematosus, these differences were not statistically significant and were not the same as those reported by Goble et $\mathrm{al}^{8}$. This may be because signal-averaged electrocardiography has a greater precision than conventional electrocardiography with an estimated error of a few milliseconds.

Analyzing the signal-averaged electrocardiogram of the subgroup of women with anti-Ro/SSA antibodies, we also did not find differences in regard to the healthy control group.

When the subject of pregnancy is considered for women with systemic lupus erythematosus, the importance of the prenatal follow-up is worth noting, because women with severe diseases as such are considered high risk. Therefore, they require special attention, such as serial ultrasounds and fetal echocardiography, especially those women positive for anti-Ro/SSA antibodies after the 16th gestational week, when the risks of neonatal lupus are greater ${ }^{15}$. Currently, no doubts exist that a well-conducted prenatal follow-up largely reduces fetal and perinatal mortality. It is also important to inform the pediatrician about possible delivery complications, allowing him to prepare appropriate material for assisting the newborn still in the delivery room. This is a determining factor for the good evolution of patients at risk, such as those with congenital total atrioventricular block. From the moment when this study was carried out until the beginning of 2002, 6 cases of congenital total atrioventricular block were diagnosed in the prenatal follow-up, although they were not included in this study. The early diagnosis allowed the successful birth of 4 children, 2 of whom underwent definitive pacemaker implantation within their first 48 hours of life.

In the sample studied, no change in ventricular function or in conduction of the cardiac stimulus was observed in children of women with systemic lupus erythematosus. Therefore, no evidence existed confirming lesions in the conduction system of these patients or factors interfering with the ventricular function in the absence of congenital total atrioventricular block, even when assessing the group of women with anti-Ro/SSA antibodies alone. Patients with no congenital total atrioventricular block may not have undergone intrauterine myocardial injury, or if they had an inflammatory process, this may have been successfully resolved without any sequelae still during the intrauterine life.

\section{References}

1. Buyon JP, Winchester R. Congenital complete heart block. A human model of passively acquired autoimmune injury. Arthritis Rheum 1990;5:33:609-14.

2. Carrera PE, Gutierrez-Larraya F, Gomez-Reino JJ. Successful intrauterine therapy with dexamethasone for fetal myocarditis and heart block in a woman with systemic lupus erythematosus. J Rheumatol 1993; 20:1204-7.

3. Tseng CE, Buyon JP. Neonatal lupus syndromes. Rheum Dis Clin North Am 1997; 23:31-53.

4. Alexander E, Buyon JP, Provost TT, et al. Anti-Ro/SSA antibodies in the pathophysiology of congenital heart block in neonatal lupus syndrome, an experimental model. In Vitro electrophysiological and immunocytochemical studies. Arthritis Rheum 1992;35:176-89.

5. Talbott JH, Historical background of discoid and systemic lupus erythematosus In: Dubois', Lupus Erythematosus. $3^{\text {rd }}$ ed. Philadelphia. London: Lea \& Febiger, 1987; 3-11.

6. Mintz G, Niz J, Gutierrez G, et al. Prospective study of pregnancy in systemic lupus erythematosus: results of a multidisciplinary approach. J Rheumatol 1986;13:732-9

7. Frohn-Mulder IM, Meilof JF, Szatmari A, et al. Clinical significance of maternal anti-Ro/SSA antibodies in children with isolated heart block. J Am Coll Cardiol 1994; 23:1677-81

8. Goble MM, Dick II M, McCune WJ, et al. Atrioventricular conduction in children of women with systemic lupus erythematosus. Am J Cardiol 1993;71:94-8.

9. Gordon PA, Khamashta MA, Hughes GR, Rosenthal E. Increase in the heart rate- corrected QT interval in children of anti-Ro positive mothers, with a further increase in those with siblings with congenital heart block: comment on the article by Cimaz et al. Arthritis Rheum 2001; 44: 242-3.

10. Cimaz R, Stramba-Badiale M, Bucrato A, Catelli L, Panceri P, Meroni PL. QT interval prolongation in asymptomatic anti-SSA/Ro - positive infants without congenital heart block. Arthritis Rheum 2000; 43:1049-53.

11. Tan EM, Cohen AS, Fries JF, et al. The 1982 revised criteria for the classification of systemic lupus erythematosus. Arthritis Rheum 1982; 25: 1271-7.

12. Sahn DJ, DeMaria A, Kisslo J, Weyman AL. The Committee on M-mode standardization of the American Society of echocardiography. Recommendations regarding quantitation in M-mode echocardiography: Results of survey on echocardiographic measurements. Circulation 1978; 58:1072-83.

13. Devereux RBH, Alfonso DT, Lutas EM, et al. Echocardiographic assessment of left ventricular hypertrophy comparison to necropsy findings. Am J Cardiol 1986; 57:450-8.

14. Waltuch J,Buyon JP. Autoantibody-associated congenital heart block: outcome in mothers and children. Ann Intern Med 1994;120:544-51.

15. Kuramochi Y, Takechi N, Ohkubo T, Ogawa S. Longitudinal estimation of signal-averaged electorcardiogram in patiens with Kawasaki disease. Pediatr Int 2002; 44: 12-7.

16. Leite MFMP, Barbosa EC, Barbosa P, et al. O eletrocardiograma de alta resolução aplicado em recém-natos. Arq Bras Ped 1997; 4 (supl. 1):27. 